\title{
Creating Embedded Platforms with MDA: Where's the Sweet Spot?
}

\author{
Tim Trew \\ NXP Semiconductors, Eindhoven, The Netherlands \\ Tim.Trew@nxp.com
}

\begin{abstract}
MDA is often promoted to enable the portability of applications across platforms, but what of the development of platforms themselves? Today's consumer electronics products, such as TVs and mobile phones are based on complex integrated circuits to support their many functions. Cost and power consumption is crucial and semiconductor suppliers have to deliver substantial amounts of software to deliver the best performance from their hardware designs. This low-level software might be exposed to customers as device drivers for the operating system of their choice, and their development gives a different perspective on "platform specific models". Given the sensitivity of this software to changes in both customer requirements and their overall software architecture, and to changes in the underlying hardware, software development and maintenance effort becomes an increasing proportion of the overall engineering effort. The presentation will describe the software development challenges faced by a semiconductor supplier and some of our experiments in meeting these challenges with MDA. It will reveal the technical limitations encountered and the greater problems of deploying unfamiliar technology across large development teams. This guides us in the hunt for the "sweet spot", where MDA delivers benefits with an acceptable learning curve and project risk.
\end{abstract}

